\title{
Investigación en modo 3: Una alternativa para la articulación investigación e intervención en educación superior
}

\author{
Claudia P. Roa-Mendoza' \\ Universidad de La Salle (Colombia)
}

Recibido: junio 14 de 2016. Revisado: julio 7 de 2016. Aceptado: agosto 24 de 2016

Referencia formato APA: Roa-Mendoza, C. P. (2016). Investigación en modo 3: Una alternativa para la articulación investigación e intervención en educación superior. Rev. Guillermo de Ockham, 14(2), 103-110. doi: http://dx.doi.org/10.21500/22563202.2635

\section{Resumen}

Las funciones sustantivas de la educación superior, a saber, docencia, investigación y extensión, requieren hoy formas que les permitan crear vínculos que faciliten su integración no solo con campos disciplinares y profesionales, sino además con escenarios sociales con el fin de aportar respuestas a las demandas de las comunidades. Responder a estas demandas requiere no solo de las tres funciones, sino también pensar los procesos de intervención. Una alternativa para articular las funciones puede ser a partir del vínculo investigación e intervención en modo 3 de conocimiento, ya que permite, entre muchas otras cosas, ir al lugar de los acontecimientos y del conocimiento, en el cual las comunidades desarrollan su capacidad de agenciamiento y pueden liderar sus procesos de análisis y transformación social.

Palabras clave: Investigación, intervención, modo 3 de conocimiento, universidad, docencia, proyección social

\section{Mode 3 research: An alternative for the articulation, research, and intervention in higher education}

\section{Abstract}

The substantive functions of higher education: teaching, research, and social projection, require today, in ways that allow them to create links to facilitate their integration not only with disciplinary and professional fields, but also with social scenarios in order to provide responses to the demands of communities. Answering these demands requires not only of the three functions, but also thought processes of intervention. An alternative that allows joint functions, can be from link research and intervention in Mode 3 knowledge, since this allows among other things going to the place of events and knowledge, in which communities develop their capacity can lead agency and analysis processes and social transformation.

Keywords: Research, intervention, mode 3 knowledge university, teaching. social projection

* Reflexiones iniciales para la investigación doctoral (2015-2018) Desafíos a la formación profesional en ciencias sociales desde las problemáticas sociales coyunturales, en el Doctorado en Educación y Sociedad de la Universidad de La Salle, línea de investigación "Saber educativo, pedagógico y didáctico".

1. Doctoranda en Educación y Sociedad de la Universidad de La Salle. Magíster en Desarrollo Educativo y Social de la Universidad Pedagógica Nacional Psicóloga y especialista en Psicología Clínica de la Universidad Católica de Colombia. Licenciada en Educación Preescolar de la Universidad Pedagógica Nacional. Docente del programa de Trabajo Social, Facultad de Ciencias Económicas y Sociales, Universidad de La Salle, Bogotá, Colombia. Grupo de investigación "Trabajo social, equidad y justicia social”, del Centro de Estudios en Desarrollo y Territorio (CEDT). Correo electrónico: claroa@ unisalle.edu.co Dirección postal: Carrera 5 No. 59 A 44, Edificio Fundadores, programa de Trabajo Social. 


\section{Pesquisa em modo 3: Uma alternativa para a pesquisa e intervenção em educação superior}

Resumo

As funçôes substantivas do ensino superior, a saber, ensino, pesquisa e extensão, requerem hoje formas que lhes permitem criar vínculos que facilitar sua integração não só com campos disciplinares e profissionais mas também com cenários sociais, a fim de fornecer respostas ás demandas das comunidades. Responder a estas demandas exige não solo das três funçôes, mas também acho os processos da intervenção. Uma alternativa para articular as funçôes pode ser a partir da ligação pesquisa e intervenção em modo 3 de conhecimento, permitindo entre outras coisas ir para o lugar de eventos e conhecimentos, em que as comunidades desenvolvam a sua capacidade agenciamiento e pode levar a análise de processos e transformação social.

Palavras-chave: Pesquisa, intervenção, modo 3 de conhecimento, universidade, ensino, projeção social

\section{Intervención e investigación, una dupla por destacar}

La intervención puede definirse desde diversas perspectivas, una de las cuales es aquella que comprende dos significados:

uno restringido, que alude a la actuación profesional dirigida a objetivos concretos; el otro, global, que la interpreta como proceso que en el tiempo y meta contexto desarrolla el sistema constituido por los profesionales y las personas que motivan su trabajo (Coletti \& Linares, 1997, p. 9).

Por tanto, la intervención en el campo de las ciencias sociales puede delimitarse como el conjunto de acciones orientadas a mediar, influir y cambiar, entre otros, las condiciones que afectan a unos sujetos de forma específica. Este proceso requiere no solo asumir una postura conceptual con base en la disciplina que fundamenta la acción, sino también aclarar la postura epistemopolítica a partir de la cual se aborda el contexto teórico y situacional de la realidad, ya que esta brinda el soporte ético, ontológico y metodológico.

Las características anteriormente enunciadas también pueden describir la investigación, de modo que valdría la pena preguntarse si no se estaría hablando del mismo proceso pero nominándolo de distinta forma.

Ante esto, autores como Osorio y Jaramillo (2013), consideran que cuando se alude a investigación e intervención se plantean dos alternativas en las que

hay prioridades particulares en cada una. La primera es el conocimiento y la segunda es la transformación. Sin embargo, hay una serie de caminos intermedios que abogan por que el conocimiento tenga como propósito transformar y que parta de los sujetos mismos como agentes transformadores, como es el caso de la investigación acción participativa, legado del maestro Fals Borda. Quienes hemos transitado por ambos caminos, sabemos que tienen ritmos, énfasis y procedimientos un tanto diferentes, pero que pueden ser muy complementarios (p. 8).

Es decir, están en un interjuego cuyos procesos de investigación deben incluir la intervención para ampliar la comprensión de las realidades abordadas y hacer un aporte a las comunidades participantes. Por su parte a la intervención subyacen procesos de investigación que dan mayor fundamentación a la acción y pueden develar la postura epistemológica de quien investiga.

Los mismos Osorio y Jaramillo consideran:

La investigación es solo una de las formas de intervención social que puede hacer y de hecho hace la academia. La asesoría, la capacitación, la gestión y la planificación son formas no excluyentes sino complementarias. Estas posibilidades se ven enriquecidas desde la universidad por la diversidad de conocimientos y de disciplinas que pueden entrar a interactuar, generando con ello aprendizajes entre profesores y estudiantes desde esas diferentes disciplinas que con frecuencia no dialogan y que se ven como campos de saber absolutamente distantes e incompatibles (Osorio y Jaramillo 2013, p. 19).

Esta relación entre investigación e intervención se materializa en la suma de producción de conocimiento y aporte/transformación a las realidades sociales. Sin embargo, según como se configuren los puntos de partida o llegada nos referiríamos a modos de conocimiento que serían determinantes para establecer la manera como se conciben la realidad, la relación con las comunidades y el posible aporte. 


\section{Modos de producción de conocimiento}

Los modos de producción de conocimiento hacen referencia a las formas de creación de conocimiento que aseguran la práctica científica. Histórica y contextualmente se presentan "tendencias que suponen, no individualmente sino en su interacción y combinación, una transformación en el modo de conocimiento" (Gibbons, Limoges, Nowonty, Schwartzman, \& Trow, 1997, p. 1). Esas transformaciones pueden ser agrupadas a partir de sus características definitorias, lo que daría lugar a la clasificación de tipos de modo de conocimiento.

Así, se plantean tres modos de producción de conocimiento (Cuadro1):

Cuadro 1

Comparación modos de producción de conocimiento

\begin{tabular}{|c|c|c|c|}
\hline Aspectos / Modos & Modo 1 & Modo 2 & Modo 3 \\
\hline Emergencia histórica & $\begin{array}{l}\text { Modernidad hasta la época } \\
\text { actual. } \\
\text { Aporte para el desarrollo del } \\
\text { capitalismo industrial, siglos } \\
\text { XVIII XIX. }\end{array}$ & $\begin{array}{l}\text { Décadas finales del siglo XX hasta la época } \\
\text { actual. } \\
\text { Consolidación de una sociedad basada en la } \\
\text { creación de riqueza, en el conocimiento, en } \\
\text { la globalización y en las empresas de carácter } \\
\text { transnacional, entre otros. }\end{array}$ & Siglo XXI. \\
\hline
\end{tabular}

Condiciones epistemo- Consolidación del paradigma $\quad$ El conocimiento es solicitado para una serie políticas. newtoniano.

Producción de conocimiento para el progreso de la humanidad.

\section{de usos específicos.}

El conocimiento deja de ser un bien de la humanidad para ser un producto al alcance de quienes pueden financiarlo.
Se da en unas condiciones de crisis ecológica planetaria, críticas al uso del conocimiento en la sociedad globalizada, insuficiencia de los modelos propuestos para el aumento de la competitividad internacional.

Necesidad de vincular otros actores sociales en la investigación.

\begin{tabular}{ll}
\hline Fundamentación. & Disciplinar. \\
\hline Intereses y preguntas de & Se plantean soluciones a pro- \\
investigación. & blemas que surgen de los inte- \\
& reses de los investigadores o de \\
& las comunidades científicas.
\end{tabular}

Contextos transdisciplinares. Transcultural.

Búsqueda de contextos de aplicación: conocimiento útil para alguien.

El conocimiento se produce bajo la negociación continua de intereses de diversos actores. Las preguntas de investigación surgen de las demandas sociales. Los contextos del problema son transitorios.

\begin{tabular}{ll}
\hline $\begin{array}{l}\text { Relación con comuni- } \\
\text { dades. }\end{array}$ & $\begin{array}{l}\text { Relación con pares poco cola- } \\
\text { borativa; las comunidades son } \\
\text { objeto de investigación. }\end{array}$ \\
\hline Generalidades & $\begin{array}{l}\text { Homogeneidad. } \\
\text { Su forma de organización es }\end{array}$ \\
& jerárquica. \\
& Adhesión a las reglas y al \\
& método científico. \\
& Oferta de investigación. \\
& La validación del conocimien- \\
& to se hace por la comunidad de \\
& especialistas.
\end{tabular}

Incluyen un conjunto de participantes que colaboran sobre un problema definido en un contexto específico.

\section{Heterogeneidad}

Su forma de organización es heterárquica y transitoria.

Socialmente responsable y reflexivo. Demanda de producción de conocimiento específico.

La validación del conocimiento se asocia a to se hace por la comunidad de la rentabilidad que se puede producir al ser cambiado el mercado.

Opera en contextos de aplicación. Las preguntas de investigación surgen de las comunidades, lo cual da como resultado un trabajo más colaborativo, que participan.

Distintos actores participan y se asumen como una comunidad de saber y conocimiento e intereses en común.

Heterogeneidad, diversidad, responsabilidad social con participación colectiva. Se busca la pertinencia ética, política y social a partir de los principios de corresponsabilidad y creación del valor social compartido, redistribución del conocimiento y sobre todo una ciencia que contribuye a un mundo más equitativo, próspero y viable. nuevas preguntas, más sectores y actores La validación del conocimiento está en función de la capacidad de aportar al mejoramiento de las comunidades participantes.

\begin{tabular}{ll}
\hline Ejemplo & Investigación de orden teórica \\
& realizada para comprobar una \\
& ley física.
\end{tabular}

Investigación que incluye demandas específicas de la empresa y qué busca la solución de un problema en particular, por ejemplo cuando se establecen alianzas estratégicas con grupos de investigación de las universidades para diseñar una prueba de selección para la institución estatal X.
Investigación que parte de las comunidades, una muestra de este son los Laboratorios de paz en los cuales distintos actores trabajan de manera mancomunada para aportar a la transformación de realidades sociales.

Fuente: Elaboración propia, basada en los planteamientos de Acosta, 2016; Acosta \& Carreño, 2013; Gibbons, Limoges, Nowonty, Schwartzman, \& Trow, 1997. 
Es claro que en todos los modos de producción del conocimiento no solo se tiene en cuenta un clima de época que favorece su generación y mantenimiento, sino que además se confiere un lugar al conocimiento como factor productor de desarrollo económico en algunas sociedades. Así, vale la pena preguntarse "¿quién decide qué conocimiento se produce?, ¿quiénes intervienen o no en este proceso productivo?, ¿quién valida o desecha el conocimiento?, ¿a quién le pertenece y por tanto, quién puede usufructuarlo? (Acosta, 2015, p. 4).

Cada modo de producción de conocimiento evidencia unas apuestas conceptuales, epistemológicas, metodológicas y políticas, que sitúan a los actores, a las prácticas y a los contextos en los que se desarrollan la investigación y la intervención de una manera específica.

El modo 3 de conocimiento se destaca, entonces, por ser una apuesta de reconocimiento a las comunidades en los procesos de investigación e intervención en los cuales se materializan las demandas sociales reales, además de concretarse los desafíos impuestos a la educación y a las universidades en una sociedad como la colombiana (Unesco, 1998; Wallerstein, 1999; Ibarra, 2000; Espinal, 2006; Zemelman, 2006; Hoyos-Vásquez, 2009; Londoño, 2011 Osorio \& Jaramillo, 2013; Ramírez-Orozco, Álvarez, \& Molano, 2013; Ovalle, 2015; OECD, 2016; Londoño \& Garzón, 2015; Vesga, 2013) ${ }^{2}$

\section{Modo 3 de producción de conocimiento y las posibilidades de articulación entre la investigación y la intervención}

Si bien se considera en los modos de producción de conocimiento 1 y 2 , que entre intervención e investigación existen varios puntos en común, estos dos procesos no son lo mismo. En su origen y desarrollo comparten acciones de fundamentación teórica, epistemológica, ontológica y metodológica asociadas, pero tienen objetivos distintos.

En tanto el resultado que busca obtener la investigación está orientado a clarificar el objeto de estudio, abordarlo para comprenderlo, estudiarlo y dar cuenta de su complejidad; la intervención social tiene el acento puesto en la acción... ambas instancias pueden compartir las herramientas y procesos de acercamiento y abordaje de objeto sobre el cual se va a operar, es decir, se problematiza, se formulan hipótesis, se realizan síntesis y diagnósticos y se seleccionan estrategias. Pero, los objetivos con los se plantea este proceso son sustancialmente diferentes. Una, la investigación, tiene objetivos investigativos cuyo horizonte es conocer una realidad determinada para comprenderla y dar cuenta de la complejidad de un fenómeno (que puede derivar en la elaboración de instrumentos y estrategias de transformación de dicho fenómeno), y la otra tiene objetivos que apuntan a la transformación y la intervención directa sobre una situación problemática que requiere resolución (Pérez, 2009, p. 2).

Así, al ser los objetivos distintos, los caminos y productos de esta perspectiva también lo son. A nivel de investigación los productos estarán asociados al conocimiento, tal como se plantea en el modo 1 , o a un contexto de aplicación específico dado en una alianza temporal como es propio del modo 2.

Sin embargo, en el modo 3 las relaciones entre estos dos procesos distan de la descripción anterior, ya que al surgir la investigación de las comunidades y su agenciamiento, se inicia una espiral de interacción en la cual investigación e intervención se articulan como una fuerza potenciadora de reconocimiento y acompańamiento de, con y para las comunidades. $^{3}$

Esta relación, se ve presentada en el Cuadro 2.

Se exponen, entonces, tres posibles formas de entender la relación entre investigación e intervención, dentro de las cuales la vinculada al modo 3, muestra una articulación entre estos procesos que pueda dar una respuesta y convertirse en alternativa de cambio para las comunidades.

Lo que se pretende con esta articulación es no solo cumplir con las funciones sustantivas de la educación superior, sino además asumir los desafíos que se plantean para las universidades y que van más allá de los perfiles de formación y producción exigidos. El punto de llegada es el desarrollo de entornos de transformación, agenciamiento y empoderamiento de las comunidades como sujetos protagonistas de sus realidades y alternativas de cambio.

A nivel nacional se adelanta una serie de discusiones sobre la pertinencia y la legitimidad de la producción de conocimiento a nivel de educación superior, y en ellas se reconoce la necesidad de vincularse con las comunidades y responder a las necesidades sentidas de las realidades y no

2. Cuenta de los desafíos, pueden resumirse en lo planteado por Londoño \& Garzón (2015) cuando afirman que "Asistimos a la más violenta mercantilización de la tierra, la cultura y la vida. Los índices de pobreza, hambre y enfermedad no disminuyen en el escenario general del mundo globalizado; situación preocupante hoy, cuando la tecnología y la investigación científica podrían aportar, más que nunca, a la solución parcial de estos problemas" (p. 7)."

3. El modo 3 estaría así en sintonía con ubicar al ser humano en el centro de la acción investigativa, desde la articulación de distintos actores sociales y con un marcado compromiso ético y social que oriente el quehacer académico (Peña, Durán, \& Gutiérrez, 2015). 
Cuadro 2

Relación entre intervención e investigación en función de los modos de conocimiento

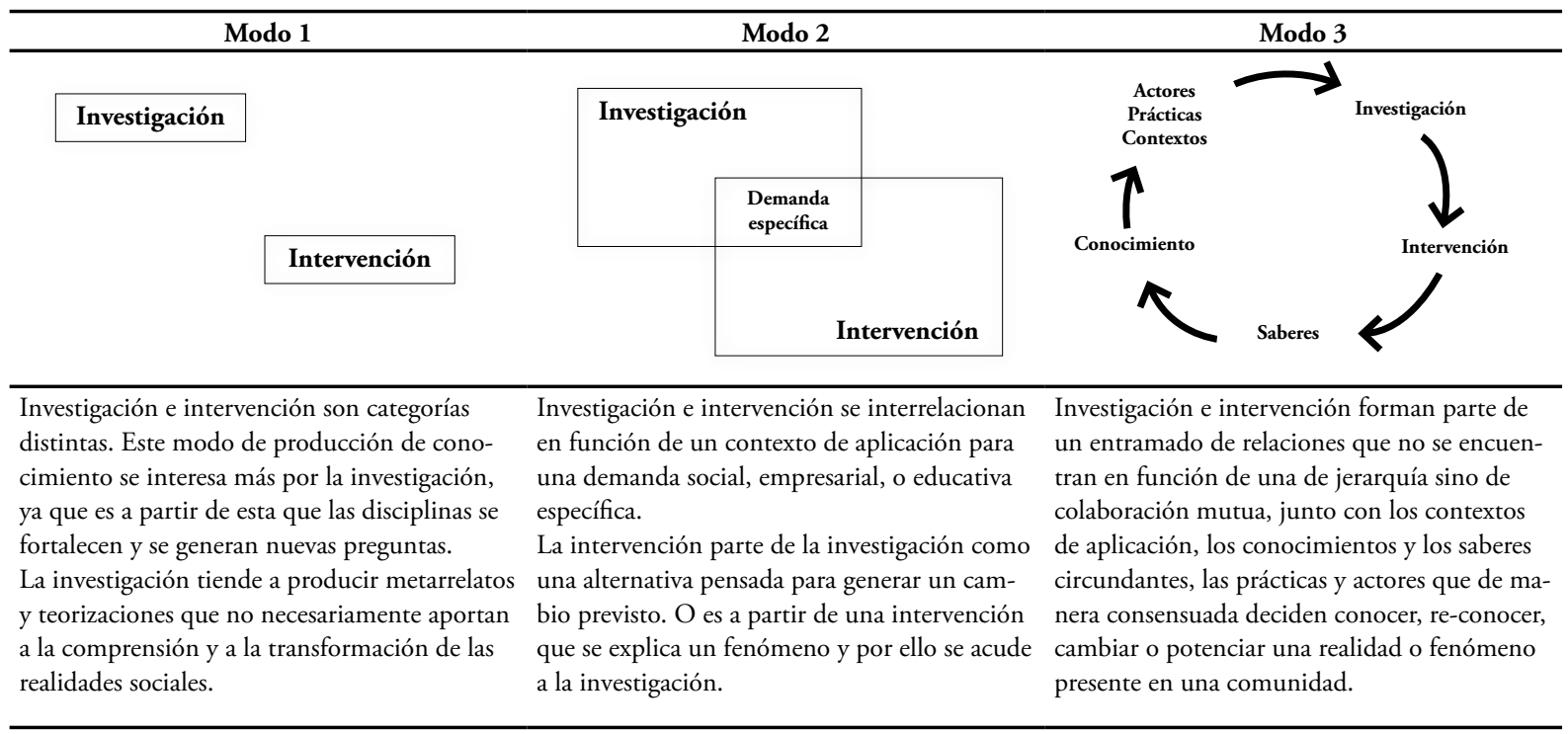

Fuente: elaboración propia.

solo circunscritos a escenarios delimitados en lo contextual y diseñados para satisfacer los campos disciplinares.

Así, "se espera que los procesos investigativos incorporen prácticas incluyentes, en donde investigadores propician la participación activa de ciudadanos y comunidades con quienes conjuntamente desarrollan iniciativas de apropiación social de la ciencia, la tecnología y la Innovación (Colciencias, 2015, p. 8).

Se reconoce que en Colciencias se presenta en ocasiones -en palabras de Ovalle (2015) - "la paradoja social", en la cual se concibe, por una parte, que la investigación e intervención pueden -y deben- aportar a la resolución de problemáticas sociales; y por la otra se considera poco científica la producción derivada de estos procesos en el campo social que, además, cuenta con un limitado presupuesto para su financiación. Debe también decirse que en los últimos años esta entidad ha circulado discursos en torno a la apropiación social del conocimiento ${ }^{4}$ y su importancia en la construcción del país.

Por tanto, se asume que

la actividad científica y creativa se debe hacer no solo en universidades e institutos de investigación, sino también en el sector productivo, de manera que exista una estrecha re- lación entre la investigación básica, la investigación aplicada y el desarrollo. Además, es fundamental que la capacidad de emprendimiento, que permite convertir esos conocimientos en innovaciones exitosas, sea parte de la formación de los profesionales, de los investigadores y de los empresarios (Colciencias, 2015, p. 2).

A lo anterior se le sumaría el contexto social con sus representantes de las comunidades, de tal manera que se transite del modo $2 \mathrm{al} \mathrm{3}$, en el cual se tenga la intencionalidad de

desestructurar las relaciones que permiten en el ejercicio intelectual reproducir las jerarquías que separan a los especialistas de sus fuentes de información, casi siempre comunidades y voces plurales. Implica romper con la escala de privilegios académicos, con la unidireccionalidad de la circulación de las ideas y con la preeminencia del capital simbólico o el estatus institucional del investigador (Osorio \& Jaramillo, 2013, p. 20).

De esta forma, se introduce el componente de colaboratividad y participación comunitaria a la articulación entre investigación e intervención. Ello se constituye en un llamado para trabajar juntos y responder a las deudas que históricamente se han reclamado a las ciencias sociales. Estas deudas no solo se inscriben en lo que plantean unos sectores de la sociedad, sino que además

4. "La apropiación social se entiende como un proceso y practica social de construcción colectiva de conocimiento, cuyos integrantes pueden ser individuos, organizaciones o comunidades, que se involucran en interacciones tendientes a intercambiar saberes y experiencias. En estos procesos el conocimiento circula, es discutido, puesto a prueba, usado y llevado a la cotidianidad, a través de estrategias de participación en las que la discusión está garantizada. De igual manera, brindan a los integrantes las herramientas para definir problemas y metodologías, plantear y probar soluciones, y tomar decisiones con base en el conocimiento elaborado y apropiado. " (Colciencias, 2015). Es decir que se estaría planteando una alternativa para materializar el Modo 3 en la articulación investigación e intervención. 
se necesita de un sistema independiente y basado en el conocimiento, que no atienda exclusivamente las demandas del gobierno de turno, sino que construya posibilidades para resolver los vacíos de conocimiento y las brechas en el desarrollo que favorecen la persistencia de las inequidades sociales (Colciencias, 2015, p. 3).

\section{Conclusiones}

Las funciones sustantivas de la educación superior, a saber, docencia, investigación y extensión, requieren hoy de formas que les permitan crear vínculos que faciliten su integración no solo con campos disciplinares y profesionales, sino también con entornos sociales con el fin de materializar los desafíos que en la actualidad se imponen a la universidad.

Una de las posibilidades para esta vinculación es la articulación investigación-intervención, ya que esta relación da cuenta tanto de la formación académica a nivel disciplinar y profesional como del retorno a los escenarios sociales de lo construido y reflexionado como respuesta a las demandas de las necesidades y problemáticas sociales.

Esta articulación se configura en distintos modos de producción de conocimiento, los cuales tienen unos intereses particulares que aterrizan en fundamentaciones teóricas, reflexiones epistemológicas y alcances metodológicos que soportan el vínculo entre academia y sociedad.

Cada modo de conocimiento, responde a un contexto histórico temporal que se asienta en una postura epistemo-política que cuenta con unos conceptos sobre el sujeto, la realidad, la naturaleza del conocimiento, sus fines y sus desafíos.

El modo 3 es una posibilidad de generar relaciones de colaboración y destacar el empoderamiento, el reconocimiento y el agenciamiento de las comunidades en la búsqueda por comprender sus propias dinámicas y apostar a alternativas de transformación social y potenciación de las capacidades actuales.

En el modo 3 se reconoce al sujeto que subyace a la investigación y la intervención, ya que pasa del sujeto receptor a un sujeto con potencial que asume una postura política y transforma su propia realidad.

Por tanto, si bien el énfasis por adoptar el modo 3 de conocimiento es una propuesta para el vínculo investigación-intervención, es también una oportunidad de fortalecimiento para las funciones de docencia y proyección social.
En particular, el modo 3 brindaría a la proyección social orientaciones para llevar a cabo un trabajo contextualizado con distintas comunidades, evidenciando con ello el carácter social de las instituciones de educación superior y las posibilidades de trabajo colaborativo para dar respuesta a las problemáticas sociales.

Lo anterior, pone sobre la mesa varios desafíos al medio universitario, como contexto de educación social que demanda cada vez más sus acciones y comprensiones, entre los cuales podrían nombrarse:

a. Las apuestas de la universidad, las cuales están relacionadas con sus objetivos fundamentales y las directrices de los estamentos reguladores de su hacer, en favor de fortalecer sus vínculos con lo social y las comunidades. Pueden encontrarse en los distintos modos de producir conocimiento, alternativas para aportar,, con base en sus proyectos educativos institucionales, a la reflexión, el reconocimiento y la transformación de las realidades sociales.

b. Los modos de producción de conocimiento se diferencian entre sí por sus aspectos epistemológicos, ontológicos y metodológicos, y son cada uno una manera de dar apertura en los campos disciplinares y profesionales a nuevas y cambiantes miradas de la realidad. En particular el modo 3, que tiene su asidero en lo social, orientan las acciones derivadas de la investigación y la intervención a las comunidades y su empoderamiento.

c. Reconocer los fenómenos sociales como dinámicos. Llama la atención sobre cuáles son los derroteros que tiene la academia para dar respuesta a las demandas de las comunidades, lo cual implica su participación activa y el reconocimiento como sujetos de cambio, materializando así el modo 3 como una alternativa de trabajo mancomunado entre la universidad y la sociedad.

d. Vincular las funciones sustantivas de la universidad a las demandas presentes en las realidades sociales, reconociendo así que estas son cambiantes y ofrecen una amplia gama de posibilidades de investigación e intervención que retroalimentan la docencia, y extensión.

e. La investigación en modo 3 de producción de conocimiento, ofrece a la academia la posibilidad de reconfigurar su acción sobre el medio social, reconociendo de esta manera la relevancia y la capacidad de agencia de las comunidades. 
f. Y finalmente, el mayor desafío, es quizás, reconocer que la transformación de la realidad social no se hace desde afuera sino desde las comunidades, lo cual implica reconocer que la academia forma parte de esa realidad, sin olvidar que no solo los escenarios cambian, sino también las problemáticas y los sujetos, por lo cual se requieren nuevas formas de comprensión y acción.

\section{Referencias}

Acosta, W. (2016). Innovación social educativa: un camino a la transformación de la realidad educativa. Bogotá.

Acosta, W. (2015). La producción de conocimiento desde la formación doctoral: elecciones y apuestas de la línea saber educativo, pedagógico y didáctico. Cuadernos de Seminario 3: Nuevas rutas para investigar la relación educación - sociedad, 3, 9-26.

Acosta, W., \& Carreño, C. (2013). Modo 3 de producción de conocimiento: implicaciones para la universidad de hoy. Revista de la Universidad de La Salle, 67-86.

Colciencias. (2015). Modelo de medición de grupos de investigación, desarrollo tecnológico o de innovación y de reconocimiento de investigadores del Sistema Nacional de Ciencia, Tecnología e Innovación, año 2015. Bogotá, Colombia: Colciencias Departamento Administrativo de Ciencia, Tecnología e Innovación. Dirección de Fomento a la investigación.

Coletti, M., \& Linares, J. (1997). La intervención sistémica en los servicios sociales ante la familia multiproblemática. Barcelona: Editorial Paidós.

Congreso de Colombia. (28 de diciembre de 1992). Ley $30 \mathrm{de}$ Diciembre 28 de 1992. Colombia.

Congreso de Colombia. (28 de diciembre de 1992). Ley 30 de Diciembre 28 de 1992. Bogotá, Colombia.

Consejo Nacional de Acreditación. (2013). Lineamientos para la acreditación de programas de pregrado. Bogotá, Colombia.

Cuadros, R. (2015). Ética y formación de investigadores: o la importancia de la sabiduría práctica. In D. Duque, J. Escobar, C. Ovalle, E. Hodson, A. Liévano, M. Guerrero, et al., Diálogos Nacionales sobre Ética de la Investigación. Memorias 2013-2014 (pp. 171-181). Bogotá, Colombia: Colciencias, Departamento Administrativo de Ciencia, Tecnología e Innovación.

Ema, J. (2004). Del sujeto a la agencia ( a través de lo político). Athenea Digital, 5, 1-24.

Espinal, C. (2006). A propósito de la universidad. Problemas, contextos y alternativas. Universitas Humanistica, 62, 91107.
Gibbons, M., Limoges, C., Nowonty, H., Schwartzman, S., \& Trow, M. (1997). La nueva producción de conocimiento. La dinámica de la ciencia y la investigación en las sociedades contemporáneas. (J. M. Pomares, Trans.) Barcelona, España: Ediciones Pomares - Corredor S.A. .

Hoyos-Vásquez, G. (2009). Educación para un nuevo humanismo. Magis, Revista Internacional de Investigación en Educación , 2, 425-433.

Ibarra, G. (2000). Las nuevas formas de producción de conocimientos y su impacto en la formación de investigadores en la UNAM. Tiempo de Educar, 2 (3-4), 66-89.

Londoño, G., \& Garzón, C. (2015). La educación rural frente a paradigmas alternativos de desarrollo: una apuesta por la esperanza. Cuadernos de Seminario 3: NUEVAS RUTAS para investigar la relación educación - sociedad, 27-46.

Londoño, G. (2011). Pertinencia del currículo crítico en el ámbito universitario. In J. Díaz, \& F. D., Curriculo y prácticas pedagógicas (pp. 105-134). Bogotá, Colombia: Ediciones Unisalle.

Ministerio de Educación Nacional. (Marzo de 2007). Glosario Educación Superior. Retrieved 24 de mayo de 2016 from Ministerio de Educación Nacional: http://www.mineducacion. gov.co/sistemasdeinformacion/1735/articles-213912_glosario.pdf

OECD. (2016). Education in Colombia. Paris: OECD Publishing.

Osorio, F., \& Jaramillo, J. (2013). Investigación e intervención social: vińetas reflexivas desde la universidad. XII Congreso "La Investigación en la Pontificia Universidad Javeriana". Bogotá: Pontificia Universidad Javeriana.

Ovalle, C. (2015). Dimensiones éticas y bioéticas de las investigaciones en Ciencias Sociales y Humanas. In D. Duque, J. Escobar, C. Ovalle, E. Hodson, A. Liévano, M. Guerrero, et al., Diálogos nacionales sobre ética de la investigación. Memorias 2013-2014 (pp. 125-143). Bogotá, Colombia: Colciencias, Departamento Administrativo de Ciencia, Tecnología e Innovación.

Ovalle, C. (2015). Dimensiones éticas y bioéticas de las investigaciones en Ciencias Sociales y Humanas. In D. Duque, J. Escobar, C. Ovalle, E. Hodson, A. Liévano, M. Guerrero, et al., Diálogos nacionales sobre ética de la investigación. Memorias 2013 - 2014 (pp. 125-143). Colciencias, Departamento Administrativo de Ciencia, Tecnología e Innovación.

Peña, C., Durán, Y., \& Gutiérrez, L. (2015). Desarrollo, educación y nueva ruralidad. Cuadernos de Seminario 3: nuevas rutas para investigar la relación educación-sociedad , 47- 62.

Pérez, M. (2009). ¿ ¿Investigar o intervenir? Aportes para un debate epistemológico en maltrato infanto-juvenil desde la perspectiva del Trabajo Social. Margen, 55. 
Ramírez-Orozco, M., Álvarez, Y., \& Molano, M. (2013). Manifiesto para pensar en borrador. In L. Pérez, Pensar en escuelas de pensamiento. Una aproximación interdisciplinar y transdisciplinar. (pp. 399-410). Bogotá, Colombia: Ediciones Unisalle.

Torres, A. (27 de abril de 2016). Metodología en ciencias sociales. Panel inaugural IV Coloquio Nacionaly II Internacional de Metodologías de la investigación Social. Investigación Socialángulos de lectura. Bogotá, Colombia: Universidad de La Salle.

Unesco . (1998). La educación superior en el siglo XXI: visión y acción. Conferencia mundial sobre la educación superior. París: Unesco.
Vesga, J. J. (2013). Cultura organizacional y sistemas de gestión de la calidad: una relación clave en la gestión de las instituciones de educación superior. Cultura organizacional y sistemas de gestión de la calidad: una relación clave en la gestión de las instituciones de educación superior. Revista Guillermo de Ockham, 11(2), 89-100.

Wallerstein, I. (1999). El fin de las certidumbres sociales. México: Universidad Autónoma de México.

Zemelman, H. (2006). El conocimiento como desafío posible. México D.F., México: Instituto Pensamiento y Cultura en América Latina, A.C. .

$110<$ Universidad de San Buenaventura, Cali - Colombia 